\title{
Drive Linac for the Argonne Wakefield Accelerator
}

\author{
E. Chojnacki, R. Konecny, M. Rosing, and J. Simpson \\ Argonne National Laboratory, Argonne, IL, 60439, USA
}

\section{INTRODUCTION}

The drive linac in Phase I of the Argonne Wakefield Accelerator (AWA) will be used to accelerate short duration (10 ps), high charge $(100 \mathrm{nC})$ electron bunches from $2 \mathrm{MV}$ to $20 \mathrm{MV}$ for use in a variety of wakefield acceleration and measurement studies[1]. The high charge is required since this drive bunch will generate the wakefields of interest in various test sections and their amplitudes are proportional to bunch charge. The short bunch duration is required to drive highfrequency wakefields without intra-bunch cancellation effects.

The drive linac design was a balance between having a small wake function to maintain a drive bunch energy spread of $\leq 10 \%$ and obtaining an adequate accelerating gradient of $\geq 10 \mathrm{MV} / \mathrm{m}$. This yielded a large aperture, low shunt impedance, high group velocity, L-band, standing-wave linac. Details of the design, fabrication, and testing are presented in the following.

\section{LINAC DESIGN}

\section{A. Broad Considerations}

The first consideration in the drive linac design was standing wave versus traveling wave. In a traveling-wave linac, the iris aperture has to be relatively smaall (low group velocity) to yield a high shunt impedance to provide an acceptable acceleration gradient given the available if source power of $\sim 20 \mathrm{MW}$. The small aperture, however, correspondingly causes the structure's wake function to be large, which would yield an unacceptable energy spread in the $100 \mathrm{nC}$ drive bunch. As an example, the SLAC Mark IV constant-impedance linac[2] scaled to $1.3 \mathrm{GHz}$, powered by a $5 \mu \mathrm{s}, 20 \mathrm{MW}$ source, and having a length of $15 \mathrm{~m}$ would provide an electron energy gain of $65 \mathrm{MeV}$, but would also produce a $47 \%$ energy spread from head to tail of the $100 \mathrm{nC}$ bunch. Refinements such as if pulse compression and utilizing a constant gradient structure would still result in a woefully large energy spread.

In a standing-wave linac, the iris aperture can be relatively large (high group velocity), yielding a low shunt impedance, but the rf power in the cavity will internally recirculate to a high steady state level, providing a reasonable accelerating gradient. The benefit of the large aperture is a low wake function, which will yield an acceptable energy spread in the $100 \mathrm{nC}$ drive bunch. Indeed, it is this low wake function characteristic of large-aperture standing-wave structures that make them attractive in super-conducting versions for the TESLA linear collider study[3]. Thus, a large-aperture standing-wave cavity was chosen for the drive linac of the
AWA. A brief parameter search of specific standing-wave geometries is presented in section IIB.

In other options, an external feedback rf system (ring resonator) would provide even larger power build up than a standing-wave cavity, but would also require the nearly impossible plumbing of $\sim 500 \mathrm{MW}$ of if power through various waveguide devices. Side-coupled structures were ruled out due to their relatively large wake functions and small acceleration gaps. Enlarging the aperture in a side-coupled structure to obtain an acceptable wake function would destroy the required isolation of adjacent beamline cavities.

\section{B. Parameter Search}

In choosing the frequency of operation of the drive linac, the overriding consideration was the longitudinal wakefield strength relative to the acceleration gradient. The amplitude of the fundamental-mode longitudinal wake function for a point source is given by

$$
W_{z}=\frac{E_{z}}{q}=\frac{\omega r_{s}}{2 Q\left(1-\beta_{g}\right)},
$$

where all parameters apply to the linac fundamental mode, $E_{z}$ is the longitudinal electric wakefield, $q$ is the bunch charge, $\omega$ is the wake frequency, $r_{s}$ is the shunt impedance in units of $[\Omega / \mathrm{m}], Q$ is the structure quality factor, $\beta_{g}$ is the normalized group velocity, and the units of $W_{z}$ are, say, $[\mathrm{kV} / \mathrm{m} / \mathrm{nC}]$. The factor $\left(1-\beta_{g}\right)$ in the denominator of $(1)$ is often ignored in cavities operating at cutoff or in traveling-wave linacs having very low group velocity. Since $r_{s} / Q$ is proportional to frequency, $f$, the longitudinal wake function in (1) is proportional to $f^{2}$, but the linac length is proportional to $f^{-1}$, so the wakefield energy loss per linac cell is proportional to $f$. Thus, as the linac frequency is lowered, the wakefield energy loss decreases linearly. This is indicative of the iris aperture being proportional to $f^{-1}$, and a smaller aperture (higher frequency) will "clip off" a larger fraction of the particle's radiation field to be left behind as wakes.

While a low drive linac frequency is desirable from a beam loading point of view, practical considerations regarding waveguide dimensions encourage the frequency to be $>1 \mathrm{GHz}$. Fortunately, examining a few test cases indicates that an Lband structure can just satisfy the gradient and energy spread goals, $\geq 10 \mathrm{MV} / \mathrm{m}$ and $\leq 10 \%$, respectively. Table I lists parameters of five standing-wave cavities given a $100 \mathrm{nC}$ drive bunch and $20 \mathrm{MW}$ of if power. These parameters were obtained from a variety of intermingled calculations utilizing analytic techniques, the URMEL computer code, and the KN7C computer code. Four of the five cases in Table I have $f=1.3 \mathrm{GHz}$ and the parameters that vary from case to case are the aperture radius $a$ and iris thickness $t$, as shown in Fig. 1 . 
TABLE I. Parameter scan of $\pi / 2$ standing-wave linacs powered by $20 \mathrm{MW}$ of if to accelerate a $100 \mathrm{nC}$ gaussian electron bunch having $\sigma=5 \mathrm{ps}$.

\begin{tabular}{|l|c|c|c|c|c|}
\hline \multicolumn{1}{|c|}{ Parameter } & $\begin{array}{c}\text { Large aperture, } \\
\text { thin iris }\end{array}$ & $\begin{array}{c}\text { Small aperture, } \\
\text { thin iris }\end{array}$ & $\begin{array}{c}\text { Large aperture, } \\
\text { thick iris }\end{array}$ & $\begin{array}{c}\text { Small aperture, } \\
\text { thick iris }\end{array}$ & $\begin{array}{c}\text { Large aperture, } \\
\text { thin iris }\end{array}$ \\
\hline Frequency $f(\mathrm{GHz})$ & 1.300 & 1.300 & 1.300 & 1.300 & 2.856 \\
\hline Aperture radius $a(\mathrm{~cm})$ & 5.08 & 2.0 & 5.08 & 2.0 & 2.31 \\
\hline Aperture/wall radius $a / b$ & 0.505 & 0.224 & 0.472 & 0.218 & 0.505 \\
\hline Aperture/wavelength $a / \lambda$ & 0.220 & 0.087 & 0.220 & 0.087 & 0.100 \\
\hline Iris thickness $t(\mathrm{~cm})$ & 0.5 & 0.5 & 3.50 & 3.50 & 0.228 \\
\hline Gap length $g(\mathrm{~cm})$ & 5.26 & 5.26 & 2.26 & 2.26 & 2.393 \\
\hline Cavity length $L(\mathrm{~m})$ & 2.07 & 2.07 & 2.07 & 2.07 & 0.94 \\
\hline Quality factor $Q$ & 17179 & 17884 & 10060 & 9755 & 12555 \\
\hline Group velocity $\beta_{g}$ & 0.139 & 0.014 & 0.048 & 0.001 & 0.139 \\
\hline Shunt impedance $r_{S}(\mathrm{M} \Omega / \mathrm{m})$ & 21.5 & 45.4 & 7.2 & 6.1 & 32.0 \\
\hline Wake function $W_{z}(\mathrm{kV} / \mathrm{m} / \mathrm{nC})$ & 5.9 & 10.5 & 3.1 & 2.6 & 27.0 \\
\hline HOM beam loading factor & 1.6 & 3.0 & 3.0 & 4.5 & 1.5 \\
\hline Voltage gain $V(\mathrm{MV})$ & $\mathbf{1 8 . 7}$ & $\mathbf{2 4 . 7}$ & $\mathbf{1 0 . 6}$ & 9.1 & $\mathbf{1 3 . 7}$ \\
\hline Voltage spread $\Delta \boldsymbol{V} / \boldsymbol{V}(\boldsymbol{\%})$ & $\mathbf{1 0 . 2}$ & $\mathbf{2 6 . 1}$ & $\mathbf{1 7 . 8}$ & $\mathbf{2 5 . 8}$ & $\mathbf{2 6 . 3}$ \\
\hline
\end{tabular}

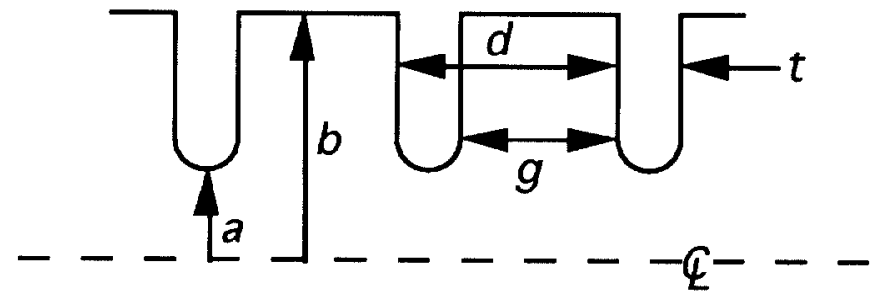

Figure 1. Schematic of standing-wave linac geometry.

It is not surprising that the thinner the iris thickness, $t$, the greater the bunch energy gain. In general, the iris thickness is made as thin as possible, limited only by mechanical strength and thermal conductivity. The fifth case in the last column of Table I is the same structure as in the first column, but scaled to $f=2.856 \mathrm{GHz}$. It is the structure in the first column with the large aperture and thin iris that was chosen for the AWA drive linac.

\section{AWA Drive Linac Properties}

The AWA drive linac is a $\pi / 2$ standing-wave linac with dimensions $a=5.08 \mathrm{~cm}, b=10.05 \mathrm{~cm}, t=0.5 \mathrm{~cm}, d=5.76 \mathrm{~cm}$, and a hemispherical aperture edge. The program $\mathrm{KN} 7 \mathrm{C}$ was used to estimate the contribution of higher order modes to the AWA drive linac axisymmetric longitudinal wakefields. Shown in Fig. 2 is a plot of the longitudinal wake left by a gaussian bunch. The fundamental wake is shown as a dashed line and the "total" wake as a solid line, which includes 42 radial modes up to a frequency of $15 \mathrm{GHz}$. The drive bunch density profiles, spaced by one rf period, are indicated on the figure as dotted lines, but only the wakefield of the first bunch is shown. From this plot, the higher order mode beam loading factor is estimated to be $\sim 1.6$.

Shown in Fig. 3 is a busy plot of the head-to-tail energy distribution that three mono-energetic electron bunches would attain upon passage through 2 meters of the AWA linac. The

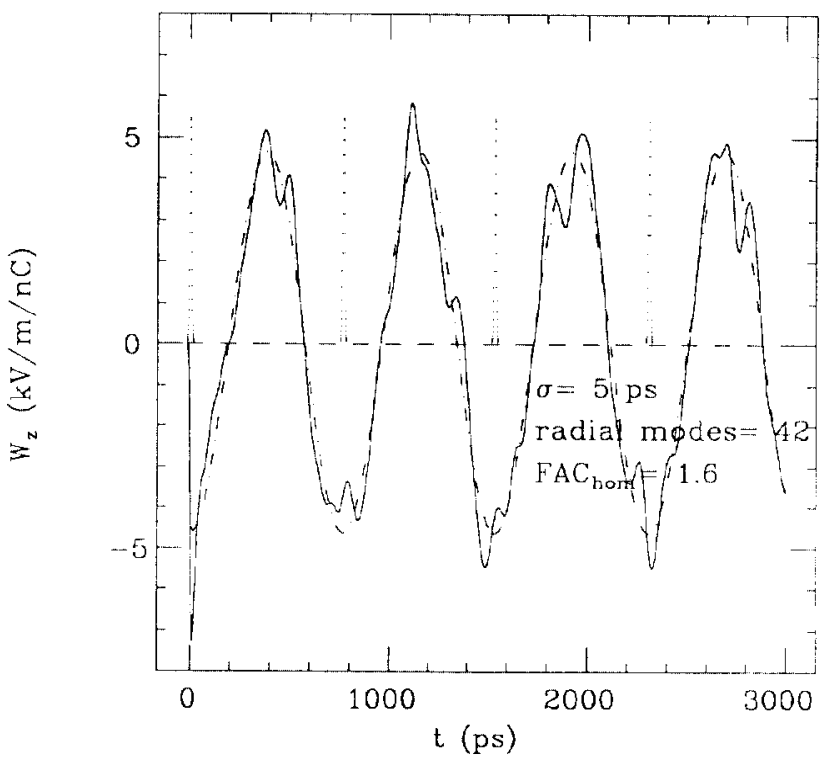

Figure 2. Plot of the longitudinal wake left by a single gaussian bunch having $\sigma=5 \mathrm{ps}$. "Total" wake is the solid line and fundamental wake is the dashed line.

higher order mode beam loading factor is included in the intrabunch energy spread, but not in the inter-bunch energy spread since the higher order modes may be somewhat out of phase for succeeding bunches. The gaussian bunch density profile is indicted as a dotted line on the first bunch's energy spread.

\section{FABRICATION}

The AWA drive linac was fabricated from OFE copper, alloy 101 , grade 2 or better, with machining of the cell cups performed on a numerical lathe. The relative machining tolerance was $\pm 0.5 \mathrm{mil}$, but the absolute tolerance was \pm 1.5 mil. Since the linac cavity frequency has to be matched 


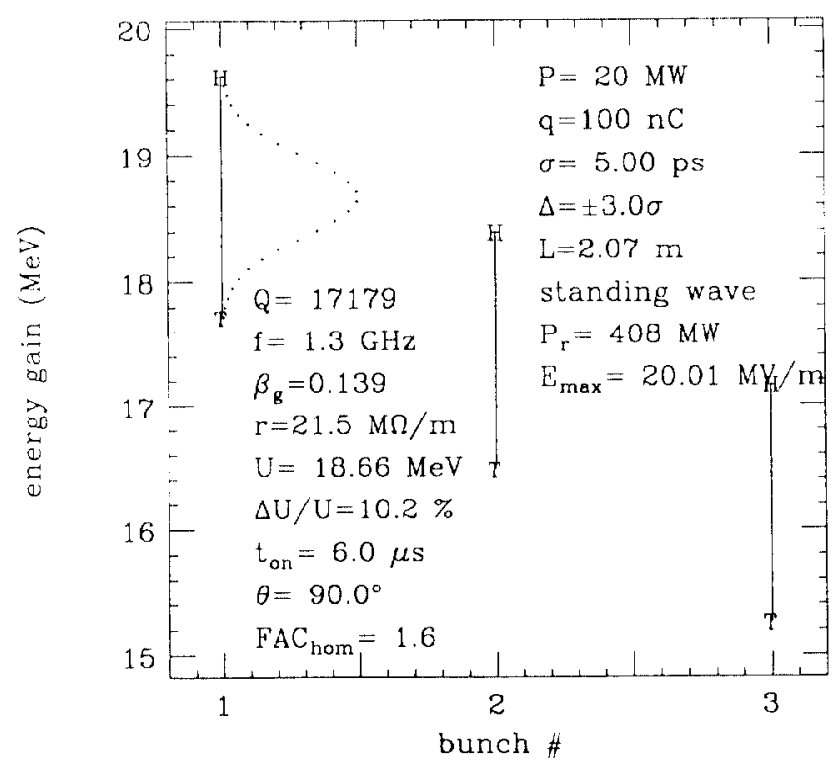

Figure 3. Plot of the head-to-tail energy distribution that three mono-energetic gaussian-profile electron bunches would altain upon passage through 2 meters of the AWA drive linac.

to the $32^{\text {nd }}$ harmonic of the AWA mode-locked laser, the absolute machining tolerance was more critical. Four 1" diameter divots leading to a $1 / 8^{\prime \prime}$ thick cell wall were machined into each cell for final frequency tuning. The rf coupling cell, which included the coupling iris and a socket for half-height Lband waveguide, was machined from a singlc block of copper. The 2 meter AWA drive linac was split into two $1 \mathrm{~m}$ tanks. The final tank assemblies were stacked vertically and brazed in a hydrogen furnace at Pyromet Industrial in San Carlos, CA.

\section{MEASUREMENTS}

Prior to brazing, the linac tanks, when clamped together, were slightly under frequency $(200 \mathrm{kHz}$ under $1.3 \mathrm{GHz}$ when corrected for temperature and vacuum conditions) and slightly under coupled to the input waveguide $\left(S_{11} \sim-15 \mathrm{~dB}\right.$ with the

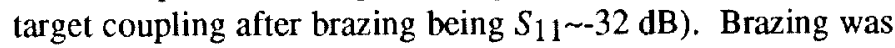
expected to improve the quality factor, $Q$, of the cavities and thus improve the coupling, and the resonant frequency was expected to rise as determined by previous brazing tests. After brazing, the resonant frequency increased only $\sim 10 \mathrm{kHz}$ and the coupling actually decreased for an unknown reason. Thus, an iterative process was implemented to improve coupling and raise the resonant frequency by enlarging the coupling iris by filing and pressing the tuning divots to raise the resonant frequency. Plotted in Fig. 4 is a sample of the latest network

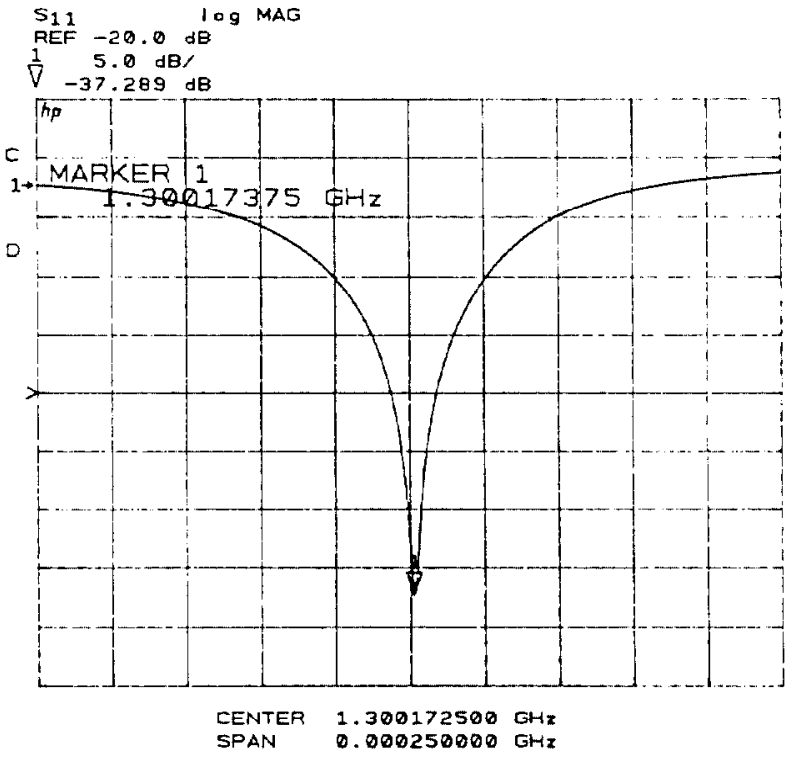

Figure 4. Network analyzer measurement of reflection coefficient $S_{11}$ versus frequency for linac tank A. Measurement performed with a $10 \mathrm{mT}$ vacuum and linac temperature of $75^{\circ} \mathrm{F}$.

analyzer measurement of reflection coefficient $S_{11}$ versus frequency for linac tank A. The coupling is satisfactory and the resonant frequency is what is needed to operate under vacuum at $\sim 90^{\circ} \mathrm{F}$. Slight final frequency tuning has yet to be performed when both tanks are connected to their temperature controlled water chillers.

This work has been supported by the United States Department of Energy, Division of High Energy Physics.

\section{REFERENCES}

[1] M. Rosing, et. al., "Argonne Wakefield Accelerator Update '92", Proc. 1992 Linear Accelerator Conf. (Ottawa, Ontario, Canada, 1992), p. 79; P. Schoessow, et. al., these proceedings.

[2] G. Loew, in The Stanford Two-Mile Accelerator, edited by R.B. Neal (W.A. Benjamin, Inc., New York, 1968), p. 129.

[3] J. Rossbach, "An Overview of Linear Collider Plans", Proc. 1992 Linear Accelerator Conf. (Ottawa, Ontario, Canada, 1992), p. 25. 